\title{
Uso de Placebo em Experimentos Clínicos Envolvendo Seres Humanos No Brasil
}

\author{
USE OF PLACEBO IN CLINICAL EXPERIMENTS \\ INVOLVING HUMAN BEINGS IN BRAZIL
}

\author{
Cristina Esteves Jordão ${ }^{(*)}$ \\ Poliana Reche Mêdola ${ }^{(* *)}$ \\ Luciane Cruz, Lopes ${ }^{(* * *)}$ \\ Pedro Luis Rosalen ${ }^{(* * * *)}$
}

\section{RESUMO}

O uso do placebo em pesquisa clínica desperta controvérsias especialmente em estudos envolvendo seres humanos. O propósito deste trabaIho é analisar o emprego ético de grupo controle-placebo e a proteção dos sujeitos da pesquisa em estudos clínicos realizados no Brasil, desde a Resolução do Conselho Nacional de Saúde CNS 196/96. Fez-se revisão sistemática (1997 a 2006) nas bases de dados Lilacs, Medline e SciELO, de estudos clínicos realizados no país com o uso de placebo e analisou-se sua natureza e aceitação ética, tendo em vista as disposições da referida Resolução.

\section{Palavras-chave}

Brasil; Placebo; Seres Humanos.

${ }^{*}$ ) Analista de Controle de Qualidade, farmacêutica e bioquímica com pós-graduação em Farmacologia Clínica na Universidade Metodista de Piracicaba. E-mail: <krikaej@yahoo.com.br>.

$\left({ }^{* *}\right)$ Coordenadora de Estudos, Centro de Estudos e Pesquisas Oncológicas de Sorocaba CEPOS SP, farmacêutica e bioquímica com pós-graduação em Farmacologia Clínica da Universidade Metodista de Piracicaba. E-mail: <polimedola@ hotmail.com>.

$\left.{ }^{* * *}\right)$ Coordenadora de curso de pós-graduação em Farmacologia Clínica na ASSPE, UNIMEP e UNISO. Email: <luslopes@terra.com.br>.

$\left({ }^{* * *}\right)$ Professor titular do Departamento de Ciências Fisiológicas da Faculdade de Odontologia de Piracicaba da Universidade Estadual de Campinas - UNICAMP. E-mail: <rosalen@ fop.unicamp>. Recebido em 9.4.08. Aprovado em 13.5.08. 


\begin{abstract}
The use of placebo in clinical research is controversy especially in studies involving human beings. The purpose of this study is to analyze the employment of ethical placebo-control groups and the protection of human beings in clinical studies in Brazil, since the Resolution CNS n. 196/96 (National Health Council). It was made a systematic review (1997 to 2006) in Lilacs, Medline and SciELO databases of clinical studies with used placebo and it was analyzed their principles and ethical acceptation, considering the mentioned resolution.
\end{abstract}

\title{
Keywords
}

Brazil; Human Being; Placebo.

\section{INTRODUÇÃO}

Desde o Código de Nuremberg(1), iniciou-se uma reflexão sobre a necessidade de se estabelecer limites com diretrizes morais, legais e éticas em experimentos envolvendo seres humanos, quase a totalidade dos documentos, internacionais ou nacionais, enfatiza a proteção do sujeito no plano de pesquisa especialmente na área médica, com a finalidade de impedir abusos em pesquisas desta natureza(2).

Posteriormente, com a Declaração de Helsinque ${ }^{(3)}$ outros documentos de natureza ética, assim como revisões da própria Declaração pela Associação Médica Mundial, foram produzidos para a melhoria da qualidade de vida, proteção e dignidade de sujeitos de pesquisas médicas ${ }^{(4)}$.

Neste sentido, o Conselho Nacional de Saúde aprovou em 10 de outubro de 1996 a Resolução CNS n. 196 ${ }^{(5)}$, sobre ética em pesquisa envolvendo

(1) TRIALS of war criminals before the Nuremberg military tribunals under Control Council Law n. 10. Washington: Government Printing Office, v. 2, p.181-182, 1949.

(2) FORTES, P. A. C. As pesquisas em seres humanos. Cadernos de Ética em Pesquisa, Brasília, v.1, n. 2, p. 22, 1998.

(3) WORLD MEDICAL ASSOCIATION. Declaration of Helsinki. Recommendation guiding physicians in biomedical research involving humans subjects. JAMA, Chicago, v. 277, n.11, p. 925-926, Mar. 1997.

(4) SARDENBERG, T. et al. Análise dos aspectos éticos da pesquisa em seres humanos contidos nas Instruções aos autores de 139 revistas científicas brasileiras. Revista da Associação Médica Brasileira, São Paulo, v. 45, n. 4, p. 295-302, 1999.

(5) BRASIL. Ministério da Saúde. Conselho Nacional de Saúde. Resolução n. 196/1996, de 10 de outubro de 1996. Diretrizes e normas regulamentadoras sobre pesquisa envolvendo seres humanos. Brasília: Conselho Nacional de Saúde, 1996. Disponível em: <http://conselho.saude.gov.br/comissao/conep/resolucao.html>. Acesso em: 20 nov. 2007. 
seres humanos, atualizada, e que atende às necessidades de evolução da ciência e tecnologia do país e principalmente garante a proteção e dignidade aos sujeitos da pesquisa. Nela se definem referências éticas (de reflexão e diretrizes quanto ao julgamento de valores, para o responsável da pesquisa, a instituição e o patrocinador), assegurando o compromisso ético e os direitos do sujeito da pesquisa. Com o objetivo de fazer cumprir essas normas foram criados o Conselho Nacional de Ética em Pesquisa - CONEP e os Comitês de Ética em Pesquisa - CEP, para funcionarem como controle social do sistema de avaliação de pesquisa. Em 2004, o país tinha 415 CEP sob cadastro no sistema ${ }^{(6)}$.

Entre os aspectos éticos da Resolução CNS n. 196/96, a restrição para o uso de placebo em pesquisas envolvendo seres humanos é de "ter plenamente justificada, quando for o caso, a utilização de placebo, em termos de não maleficência e de necessidade metodológica", item III.3f ${ }^{(7)}$.

A Declaração de Helsinque ${ }^{(8)}$ define a utilização em qualquer estudo médico do melhor método existente de diagnóstico e terapêutica para cada paciente, incluindo os do grupo-controle, se houver esse grupo no estudo, não excluindo o uso de placebos inertes em estudos nos quais não existam métodos diagnósticos ou terapêuticos comprovados.

Em 2002, fez-se uma Nota de Esclarecimento sobre o Parágrafo 29, à revisão da Declaração de Helsinque da $52^{-a}$ Assembléia Geral da AMM (Associação Médica Mundial), Anexo II, referente ao uso de placebo no qual constam os cuidados extremos com o uso do placebo, que, em geral, só pode ser usado no caso de inexistência de terapia, além de haver necessidade de uma apropriada revisão ética e científica do estudo. Nesta nota, consta ainda que pesquisas são eticamente aceitáveis por sérias razões metodológicas e científicas para determinar a eficácia ou a segurança de um método profilático, diagnóstico ou terapêutico ou quando um método profilático, diagnóstico ou terapêutico estiver sendo pesquisado por condição irrelevante e que não oferece risco adicional de danos sérios e irreversíveis aos pacientes a administração do placebo $^{(9)}$.

Há importância no uso do placebo em pesquisas de novos medicamentos, principalmente pelo interesse econômico e de desenvolvimento das indústrias farmacêuticas. Porém, justifica-se o uso de placebo na compara-

(6) FREITAS, C. B. D.; LOBO, M.; HOSSNE, W. S. Oito anos de evolução: um balanço do Sistema CEP-CONEP. Cadernos de Ética em Pesquisa, Brasília, v. 6, n. 16, p. 20-30, 2005. Disponível em: <http://conselho.saude.gov.br/comissao/conep/publicacoes_cep.html>. Acesso em: 20 nov. 2007. (7) BRASIL. Ministério da Saúde. Conselho Nacional de Saúde. Resolução n. 196/1996, de 10 de outubro de 1996. Diretrizes e normas regulamentadoras sobre pesquisa envolvendo seres humanos, cit. (8) WORLD MEDICAL ASSOCIATION. Declaration of Helsinki. Recommendation guiding physicians in biomedical research involving humans subjects, cit.

(9) OLIVEIRA, G. G. Ensaios clínicos: princípios e práticas. Brasília: Ministério da Saúde, 2006. p. 255-256. 
ção no tratamento com o novo fármaco, se não houver tratamento mínimo e de eficácia comprovada para a doença ${ }^{(10)}$.

Os avanços na área de pesquisa clínica estão cada vez mais evidentes, a ética e o sujeito de pesquisa são assuntos discutidos em universidades e instituições públicas e privadas, já existindo casos nos quais o CONEP não aprova pesquisas clínicas, onde o medicamento em teste for comparado com um placebo, sendo o grupo controle tratado com outro medicamento já utilizado, permitindo que o sujeito de pesquisa receba tratamento(11).

Diante dessas disposições, este estudo visa verificar se, na vigência da Resolução CNS n. 196/96 ${ }^{(12)}$, pesquisas brasileiras editadas que envolveram o uso de placebo têm aplicado a legislação na avaliação e no julgamento de valores.

\section{MATERIAIS E MÉTODOS}

Publicações de artigos científicos completos de estudos clínicos no Brasil, de janeiro de 1997 a janeiro de 2006, foram localizadas por meio das seguintes bases de dados: SciELO (SciELO - Scientific Electronic Library Online em http:// www.SciELO.br), Medline (base de dados criada e mantida pela National Library of Medicine - NLM, em http://www.ncbi.nlm.nih.gov) e Lilacs (Literatura LatinoAmericana e do Caribe em Ciências da Saúde em https://www.bireme.br).

Os descritores utilizados foram: "placebo", "seres humanos" ("ser humano" ou "humans") e "Brasil" (ou Brazil).

De acordo com as referências bioéticas como: autonomia, beneficência, não-maleficência e justiça, constantes na Resolução CNS n. 196/96(16), foram avaliados os artigos selecionados. Igualmente, utilizando-se como base o item III da Resolução citada que abrange a ética no uso de placebos, os artigos foram avaliados segundo aspectos envolvendo: a) objeto do estudo; b) tipo de estudo realizado e efeito placebo; c) aprovação por Comitê de Ética; d) sujeito da pesquisa; e e) alegações que justificam o uso do placebo.

Verificou-se o objeto de cada estudo, categorizando-os em "farmácos novos", "fármacos já registrados, fase 4" e "fármacos registrados, mas para uso diferente do registro". Foram consideradas as áreas de pesquisa, as áreas temáticas (Resolução CNS n. 196/96, VIII-4), o tipo de estudo realizado (multicêntrico, cego, duplo-cego, simples-cego, randomizado, assim como a presença de efeito placebo nos resultados obtidos) $)^{(13)}$.

(10) COMISSÃO NACIONAL DE ÉTICA EM PESQUISA. A CONEP responde: Três questões básicas. Cadernos de Ética em Pesquisa, Brasília, v. 1, n. 2, p. 4-6, 1998.

(11) PINTO M. Relatório setorial final: setor farmacêutico — Lab. Nacionais. Rede DPP FINEP, 2004. Disponível em: <http://www.finep.gov.br/PortalDPP>. Acesso em: 20 abr. 2007.

(12) BRASIL. Ministério da Saúde. Conselho Nacional de Saúde. Resolução n. 196/1996, de 10 de outubro de 1996. Diretrizes e normas regulamentadoras sobre pesquisa envolvendo seres humanos, cit. (13) Id. Ibid. 
Os artigos foram agrupados em relação àqueles que informaram se a pesquisa foi aprovada por Comitê de Ética e selecionada considerando o ano de edição. Quanto à proteção do sujeito de pesquisa, observou-se se o artigo informou o uso do Termo de Consentimento Livre e Esclarecido ou equivalente e se houve tratamento de recuperação (medicação de resgate).

A justificativa do uso do placebo foi definida conforme as diretrizes acima assinaladas, caso esta informação não constasse de forma clara no artigo.

Os critérios de exclusão aplicados na estratégia de busca eletrônica e manual dos artigos foram: 1) artigos de revisão bibliográfica; 2) artigos de estudos que não envolviam seres humanos; 3) artigos que mencionavam o Brasil, mas o estudo não ocorreu no país; 4) artigos com mais de um registro nas bases de dados e, nestes casos, utilizou-se a base de dados Lilacs como referência, pois se trata de uma base do Sistema BIREME e que compreende a literatura relativa às Ciências da Saúde, incluindo a Literatura Latino-Americana e do Caribe ${ }^{(14)}$; e 5) artigos incompletos que não puderam ser acessados eletronicamente ou pelo sistema de comutação de artigos entre bibliotecas.

\section{RESULTADOS E DISCUSSÃO}

Os resultados das buscas nas três bases de dados utilizando-se os descritores informados permitiram a localização de 218 artigos científicos completos (Tabela 1), envolvendo estudos clínicos com emprego de grupo controle-placebo e realizados no Brasil, indexados no período de janeiro de 1997 a janeiro de 2006.

Tabela 1. Número de artigos indexados em bases de dados de estudos clínicos com emprego de grupo controle-placebo no Brasil; janeiro de 1997 a janeiro de 2006

\begin{tabular}{|c|c|c|c|c|}
\hline $\begin{array}{c}\text { Base de } \\
\text { dados }\end{array}$ & $\begin{array}{c}\text { Total de } \\
\text { artigos (1) }\end{array}$ & $\begin{array}{c}\text { Total de } \\
\text { artigos com } \\
\text { descritores (2) }\end{array}$ & $\begin{array}{c}\text { Artigos } \\
\text { incluídos (n) }\end{array}$ & $\begin{array}{c}\text { Percentual de } \\
\text { incluídos (\%) }\end{array}$ \\
\hline Lilacs & 1541 & 444 & 152 & 69,7 \\
Medline & 115.584 & 192 & 64 & 29,4 \\
SciELO & 214 & 61 & 02 & 0,9 \\
\hline Total & 116.633 & 697 & 218 & 100 \\
\hline
\end{tabular}

NOTA: (1) Descritor placebo. (2) Descritores: placebo, Brasil ou Brazil e ser humano ou human.

(14) BIREME/OPAS/OMS. Biblioteca Virtual em Saúde e o controle bibliográfico da produção científica da América Latina e Caribe: o sistema e bases de dados LILACS. São Paulo: Bireme, 2006. Disponível em: <http://bvsmodelo.bvsalud.org/site/Lilacs>. Acesso em: 30 maio 2006. 
A escolha das bases bibliográficas Lilacs, Medline e SciELO para este trabalho deveu-se à abrangência nacional e internacional na área da saúde destas bases.

Observa-se na Tabela 1 que a maioria dos artigos foram divulgados na base de dados Lilacs (69,7\%), sendo assim considerada a base de referência. O Brasil é maior colaborador da Lilacs, tendo contribuído com média ponderada anual de 13.366 registros, o que representa $52,6 \%$ da contribuição total e, em 2005, a contribuição do país chegou a 63,2\%. A Lilacs constitui-se no principal índice bibliográfico da Biblioteca Virtual de Saúde (BVS), fato que se justifica por estar voltada para a divulgação da literatura científica produzida na América Latina e no Caribe na área de Ciências da Saúde. Desde sua criação em 1982, vem ampliando significantemente o número de periódicos e outros documentos indexados. Algumas revistas indexadas na Lilacs estão disponíveis em texto eletrônico na SciELO, havendo assim registros múltiplos de 59 artigos do total de $61^{(15)}$.

A base de dados Medline contém citações bibliográficas e resumos de artigos editados em mais de 4.000 revistas biomédicas dos Estados Unidos e de 70 países, com mais de 10 milhões de referências bibliográficas, desde $1966^{(16)}$. Esta base representou neste levantamento $29,4 \%$ dos artigos avaliados, dos quais, $19,3 \%$ (37 artigos do total de 192) eram artigos também registrados na base de dados Lilacs. Por outro lado, 31 artigos da Lilacs foram identificados na base Medline. A Bireme colabora com a base de dados Medline, indexando artigos. Em 2006, foram 1.640 artigos de revistas para esta base de uma média mensal de 328 artigos ${ }^{(17)}$.

As bases de dados revelam, segundo o primeiro descritor "placebo", um número de registros elevado, 116.633 artigos. No entanto, quando se adiciona o segundo descritor "Brasil" ou "Brazil", o número total reduz consideravelmente para 697 artigos (Tabela 1). Os limites definidos, assim como os critérios estabelecidos no estudo, delimitaram a busca e apontaram que alguns estudos foram realizados no Brasil, mas, por serem pesquisas multicêntricas, os dados não foram somados aos estudos no país de origem. Ademais, por não estar explícito em alguns artigos que o Brasil teve participação no experimento não foram considerados no trabalho.

Dos 142 registros bibliográficos que não tinham disponibilidade eletrônica na íntegra ou pelo sistema de comutação de artigos entre bibliotecas, 83 artigos eram teses de doutorado ou mestrado, o que impediu a inclusão neste trabalho.

(15) PINTO M., op. cit.

(16) PELLIZZON, R. F.; POBLACIÓN, D. A.; GOLDENBERG, S. Pesquisa na área da saúde: seleção das principais fontes para acesso à literatura científica. Acta Cirurgica Brasileira, v.18, n. 6, 2003. (17) BIREME/OPAS/OMS. Biblioteca Virtual em Saúde e o controle bibliográfico da produção científica da América Latina e Caribe: o sistema e bases de dados LILACS, cit. 
A quantidade total de artigos incluídos de acordo o período estabelecido é pequena (218), considerando que o índice anual de crescimento de pesquisa clínica no período de 1997 a 2001 foi superior a 10\% ao ano, segundo a Agência Nacional de Vigilância Sanitária (ANVISA - 2003). Além disso, somente em $2002^{(18)}$, 846 projetos foram aprovados pelo CONEP. Observou-se que o número de pesquisas realizadas no período de 1999 a 2000 cresceu $71 \%$, segundo o CONEP com o início do controle social definido pela Resolução CNS n. 196/96(19); mas, esses números não refletem na produção científica nacional avaliada nesse estudo com base nos limites selecionados.

Em outubro de 2005, a Coordenação de Aperfeiçoamento de Pessoal de Ensino Superior (Capes) divulgou dados sobre a produção científica brasileira que cresceu 15\% em 2004. E, segundo o documento, quanto à importância das pesquisas clínicas feitas no país, os cientistas garantem que o aumento não foi apenas quantitativo, mas, também, qualitativo(20).

O crescimento científico foi também confirmado por edições realizadas por especialistas como o Institute for Scientific Information (ISI, Filadélfia, EUA):(21)

Entre os 30 países mais destacados no ranking da ciência mundial os quais são responsáveis por $90 \%$ dessa produção, o crescimento do Brasil, em tão curto espaço de tempo, só foi menor do que o de alguns poucos países desse conjunto, que também tiveram crescimento excepcional: Coréia do Sul, Taiwan, China, Espanha e Turquia.

Verificou-se pelo levantamento apresentado que no período de janeiro de 1997 a janeiro de 2006 o crescimento da produção científica nacional foi intenso; porém, não foi possível confirmá-lo por meio dos descritores e limites utilizados nessa pesquisa, portanto, a produção científica nacional não está nítida neste estudo.

Uma das razões para explicar o crescimento da produção científica brasileira é a constância de investimentos internos em pesquisa e desenvolvimento nos últimos anos e também o aperfeiçoamento das instituições de pesquisa, além de mudanças decorrentes de fusões políticas adotadas pe-

(18) VILARDAGA, V. Autorização para testes clínicos com brasileiros cresce $10 \%$ ao ano. Revista Pesquisa FAPESP, São Paulo, n. 83, jan. 2003. Disponível em: <http://www.revistapesquisa.fapesp.br>. Acesso em: 20 abr. 2007.

(19) LEITE, F.; SILVA, J. C. Em nome da ciência: Brasil já tem 500 mil "cobaias" humanas. Folha de S. Paulo, São Paulo, 29 jun. 2001.

(20) ACADEMIA BRASILEIRA DE REUMATOLOGIA. A Produção Científica Brasileira. Boletim Acadêmico - ABR, Recife, v. 6, n. 1, p. 4-5, jan./mar. 2006. Disponível em: <http://www.academiareumatol.com.br/ boletim/boletim11.pdf>. Acesso em: 20 nov. 2007.

(21) GUIMARÃES, J. A. A pesquisa médica e biomédica no Brasil: comparações com o desempenho científico brasileiro e mundial. Ciência \& Saúde Coletiva, Rio de Janeiro, v. 9, n. 2, p. 303-327, 2004. 
las fundações nacionais como o Conselho Nacional de Desenvolvimento Científico e Tecnológico - CNPq e a Capes $^{(22)}$.

Entre os artigos analisados, destacam-se alguns dados, especialmente o ano de edição e as regiões do Brasil onde foram realizados os estudos. Notou-se que $61,0 \%$ das publicações concentraram-se de 2002 a 2005. Além disso, 37,6\% das pesquisas foram realizadas nos Estados de São Paulo e do Rio de Janeiro. Na região Sudeste, concentra-se a maioria das universidades que desenvolvem estudos clínicos e também uma grande quantidade de CEP (241), representando 58,1\% dos Comitês do país em 2005(23).

Da análise dos dados, observou-se que a área médica é onde se reúne a maioria dos estudos, sendo que as especialidades que divulgaram pesquisas com placebo, considerando seus limites, foram as de Neurologia e Psiquiatria $(26,2 \%)$, Imunologia (9,6\%), Odontologia (7,8\%) e Ginecologia (6\%).

A avaliação quanto à presença de efeito placebo foi constatada em 8,7\% (19 edições) do total dos artigos analisados. Destes, 6 trabalhos eram da especialidade médica de Neuropsiquiatria (psicofármaco), que vem utilizando e debatendo intensamente o uso do placebo. As agências reguladoras dos Estados Unidos, do Japão e a comunidade científica internacional defendem o uso do grupo controle-placebo em pesquisas que utilizam psicofármacos, sendo considerado indispensável para a determinação de tratamentos eficazes nos transtornos mentais. A alegação para o uso do grupo controle-placebo deve-se às possíveis interferências de fatores específicos durante um estudo com outros fármacos e um psicofármaco. Porém, é necessária sempre a avaliação ética quanto ao uso, assim como um termo de consentimento livre e esclarecido, elaborado e aplicado de forma correta, garantindo-se ao sujeito da pesquisa o entendimento quanto ao seu riscobenefício(24).

Levando em consideração o item VIII-4c da Resolução CNS n. 196/96 (25) que dispõe das atribuições da CONEP em aprovar e acompanhar os protocolos de pesquisa em áreas temáticas especiais, todos os artigos foram enquadrados na área temática 3, ou seja, a maioria são estudos que envolvem fármacos, medicamentos, vacinas e testes diagnósticos novos (fases I, II e III) ou não registrados no país (ainda que na fase IV). Há ainda estudos referentes ao uso com modalidades, indicações, doses ou vias de administração diferentes daquelas estabelecidos, incluindo emprego em combinações.

(22) ZORZETTO, R. et al. The scientific production in health and biological sciences of the top 20 Brasilian Universities. Brazilian Journal of Medical and Biological Research, São Paulo, v. 39, n.12, p. 1513-1520, 2006.

(23) FREITAS, C. B. D.; LOBO, M.; HOSSNE, W. S., op. cit.

(24) VERSIANI, M. A necessidade do Grupo-Controle com placebo em pesquisas sobre a eficácia de tratamentos psiquiátricos. Revista Bioética, Brasília, v. 8, n. 1, p. 29-42, 2000.

(25) BRASIL. Ministério da Saúde. Conselho Nacional de Saúde. Resolução n. 196/1996, de 10 de outubro de 1996. Diretrizes e normas regulamentadoras sobre pesquisa envolvendo seres humanos, cit. 
$\mathrm{Na}$ Tabela, 2 apresenta-se os resultados referentes ao objeto do estudo: edições com citações quanto à "fármaco novo", "fármaco já registrado, fase 4" e "fármaco já registrado, mas, não para o fim estudado", resultando maior número de estudos com fármacos registrados $(81,2 \%)$. As pesquisas realizadas na fase 4 abrangem experimentos pós-registro do produto e/ou da especialidade medicinal, podendo ser estudos de vigilância pós-registro, para estabelecer o valor terapêutico, o surgimento de novas reações adversas e/ou confirmação da freqüência de surgimento das já conhecidas e as estratégias de tratamento, aplicando as normas éticas e científicas das demais fases ${ }^{(26)}$. Entre os fármacos registrados observou-se que $54,7 \%$ dos artigos referiam-se a estudo de comprovação terapêutica e não mencionavam outras características da fase 4 , como surgimento de reações adversas e estratégias de tratamento.

\section{Tabela 2. Número de edições com citações sobre "fármacos novos", "fármacos já registrados, fase 4" e "fármacos já registrados, mas para uso diferente do registro"}

\begin{tabular}{|l|c|c|}
\hline \multicolumn{1}{|c|}{ Objeto do estudo } & Artigos $(\boldsymbol{n})$ & Porcentual (\%) \\
\hline Fármaco novo & 25 & 11,5 \\
\hline Fármaco já registrado, fase 4 & 94 & 43,1 \\
\hline $\begin{array}{l}\text { Fármaco já registrado, mas para uso diferente do } \\
\text { registro }\end{array}$ & 83 & 38,1 \\
\hline Outros (vacinas e testes diagnósticos novos) & 16 & 7,3 \\
\hline Total & 218 & 100 \\
\hline
\end{tabular}

A Tabela 3 refere-se ao "tipo de estudo realizado", informando a prevalência de estudos "duplo-cego" (80,3\%), desejável, pois, reduz o risco de interferências ${ }^{(27)}$.

(26) BRASIL. Ministério da Saúde. Agência Nacional de Vigilância Sanitária. Considerações e definições para Pesquisa Clínica. [s.d.]. Disponível em: <http://www.anvisa.gov.br/medicamentos/ pesquisa/def.htm>. Acesso em: 20 mar. 2006.

(27) CONFERÊNCIA INTERNACIONAL DE HARMONIZAÇÃO. Manual para boas práticas clínicas: versão Harmonizada Tripartite (USA, Europa e Japão). Tradução de Marion Roussel, 1996. Disponível em: <http://www.ufrgs.br/bioetica/gcpport.htm>. Acesso em: 30 maio 2006. 
Tabela 3. Número de edições com citações sobre o tipo de estudo: cego ou duplo-cego, "randomizado" e multicêntricos

\begin{tabular}{|l|c|c|c|c|}
\hline \multicolumn{1}{|c|}{ Tipo de estudo } & Cego & Duplo-cego & Randomizado & Multicêntricos \\
\hline Artigos (n) & 13 & 175 & 205 & 27 \\
\hline Porcentual (\%) & 5,9 & 80,3 & 94,0 & 12,4 \\
\hline
\end{tabular}

Estudos controlados ao acaso (randomizados) foram descritos em $94,0 \%$ (Tabela 3) das edições, mas as pesquisas que não informaram a distribuição ao acaso, "randomização", (5,9\%) podem ter falhas, sendo ou não exigência da revista este dado para divulgação, que consta no Manual de Boas Práticas Clínicas ${ }^{(28)}$. A "randomização" é reconhecida como cientificamente desejável para se avaliar um tratamento novo, devendo ser realizada eticamente somente quando os sinais e sintomas que o sujeito de pesquisa poderia apresentar não causariam nenhum dano irreversível(29).

Os estudos multicêntricos são recomendados e de importância para a pesquisa, pois utilizando o mesmo protocolo pode-se obter resultados diversos em centros diferentes, o que é estatisticamente melhor que dados obtidos de único experimento, uma vez que se observa diversidade e maior quantidade de pacientes para o estudo(30). Porém, pode-se ver nos resultados da Tabela 3 que apenas $12,4 \%$ dos estudos são multicêntricos, enquanto $45,9 \%$ dos artigos não citam onde a pesquisa foi realizada. Os protocolos do exterior submetidos aos Comitês de Ética em Pesquisa geralmente estão prontos $^{(31)}$, o que pode ser a razão pela não inclusão do Brasil na edição da pesquisa. É importante que estudos multicêntricos internacionais informem os países participantes, fato este não considerado na legislação nacional e internacional, mas relevante para saber o que de fato ocorre no país quanto à pesquisa em seres humanos.

A aprovação do estudo por "Comitê de Ética" foi informada com frequência nas edições como consta na Tabela 4, em 75,2\% dos artigos, seguindo a exigência da Resolução CNS n. 196/96 (32), o que reflete a importância da legislação vigente e o seu cumprimento, uma vez que toda pesquisa envolvendo seres humanos deve ser submetida à apreciação de um Comitê de Ética. As edições que não citam a aprovação por Comitê de Ética, 24,8\%, podem não se enquadrar nas exigências e normas (Instruções aos autores)

(28) Op. cit.

(29) LEWIS, J. et al. Placebo-controlled trials and the Declaration of Helsinki. Lancet, London, v. 359, n. 9314, p. 1337-1340, 2002.

(30) HOSSNE, W. S.; VIEIRA, S. Pesquisas com cooperação estrangeira: qual cooperação? Cadernos de Ética em Pesquisa, Brasília, v. 6, n. 14, p. 3-5, 2004. [editorial].

(31) Id. Ibid.

(32) BRASIL. Ministério da Saúde. Conselho Nacional de Saúde. Resolução n. 196/1996, de 10 de outubro de 1996. Diretrizes e normas regulamentadoras sobre pesquisa envolvendo seres humanos, cit. 
para divulgação em revistas. Algumas delas solicitam informação sobre a aprovação por Comitê de Ética, não se exigindo que isso conste no artigo. Convém salientar que os estudos internacionais também devem ter a aprovação por Comitê de Ética, conforme a Declaração de Helsinque ${ }^{(33)}$.

Tabela 4. Número de edições com citações sobre a aprovação do estudo por Comitê de Ética e obtenção de Termo de Consentimento Livre e Esclarecido (TCLE)

\begin{tabular}{|l|c|c|}
\hline & $\begin{array}{c}\text { Informam aprovação } \\
\text { por Comitê de Ética }\end{array}$ & Obtenção de TCLE \\
\hline Artigos (n) & 164 & 163 \\
\hline Porcentual (\%) & 75,2 & 74,8 \\
\hline
\end{tabular}

Normas relativas à ética da pesquisa em seres humanos contidas nas "instruções aos autores de revistas de Ortopedia e Traumatologia" foram estudadas e observou-se que $52,6 \%$ não faziam referência a aspectos éti$\cos ^{(34)}$, assim como estudo de revistas de Medicina e Biomedicina de Língua Inglesa não apresentaram referência à ética nas "Instruções aos Autores" em $24 \%$ dos estudos(35). Na área de saúde no Brasil, 139 revistas científicas brasileiras foram analisadas e apenas 20,8\% apresentaram referências éticas nas Instruções aos Autores ${ }^{(36)}$. De acordo com esses dados conclui-se que aspectos éticos devem ser enfatizados nas "Instruções aos Autores" assim como deve ser observado por eles no momento da submissão do artigo, evitando assim que sejam divulgados de forma não ética.

Quanto ao "sujeito de pesquisa e sua proteção" foram verificados os artigos que informaram se os pacientes da pesquisa assinaram o "Termo de Consentimento Livre e Esclarecido" (TCLE) antes da participação no estudo. Verifica-se pela Tabela 4 que $74,8 \%$ das edições informaram a assinatura, exigência da Resolução CNS n. 196/96 ${ }^{(37)}$ cumprida, sendo indispensável para a realização da pesquisa.

No porcentual de artigos que informaram a assinatura do TCLE, foram consideradas as formas alternativas de obtenção do termo de consentimen-

(33) WORLD MEDICAL ASSOCIATION. Declaration of Helsinki. Recommendation guiding physicians in biomedical research involving humans subjects, cit.

(34) SARDENBERG, T. et al. Análise dos aspectos éticos da pesquisa em seres humanos contidos nas Instruções aos autores de 139 revistas científicas brasileiras, cit.

(35) AMDUR, R. J.; BIDDLE, C. Institutional review board approval and publication of human research results. JAMA, Chicago, v. 277, n. 11, p. 909-914, 1997.

(36) SARDENBERG, T. et al., op. cit., p.295-302.

(37) BRASIL. Ministério da Saúde. Conselho Nacional de Saúde. Resolução n. 196/1996, de 10 de outubro de 1996. Diretrizes e normas regulamentadoras sobre pesquisa envolvendo seres humanos, cit. 
to e entre os artigos que não informaram incluem-se os que não realizaram por se tratarem de estudos psiquiátricos autorizados pelos CEPs. Entretanto, o percentual de $25,2 \%$ é elevado para não se informar a existência de TCLE ou equivalente. Esta é uma das informações que necessita ser avaliada pelos pesquisadores e editores das publicações, apesar da maioria das "Instruções aos Autores" não exigir a declaração de TCLE.

A possibilidade de uso de medicação de resgate nos estudos está indicada em 59,2\% dos artigos (Tabela 5), mas, apenas, 25,7\% informaram o uso quando aplicado. O uso de medicação de resgate, com a finalidade de proteção ao sujeito de pesquisa ${ }^{(38)}$, pode ser necessário em caso de interrupção de tratamento por algum motivo ou de alguma reação adversa.

Tabela 5. Número de edições com citações sobre a possibilidade de uso de medicação de resgate

\begin{tabular}{|l|l|c|c|}
\hline \multirow{4}{*}{$\begin{array}{l}\text { Medicação } \\
\text { de resgate }\end{array}$} & Aplica-se & 129 & 59,2 \\
\cline { 2 - 4 } & Quando aplicável, existe informação & 56 & Porcentual(\%) \\
\cline { 2 - 4 } & Quando aplicável, não existe informação & 73 & 33,7 \\
\cline { 2 - 4 } & Não se aplica & 89 & 40,8 \\
\hline
\end{tabular}

Os sujeitos de pesquisa estavam isentos de tratamento na maioria das edições $(85 \%)$ e, em $6,4 \%$, eram voluntários sadios. Várias alterações na Declaração de Helsinque foram propostas; porém, não aceitas quanto ao uso do placebo. Em 2000, assegurou-se que "no final do estudo todos os doentes devem continuar a ter acesso aos melhores métodos de tratamento identificados no estudo"(39). Desvios nas normas preestabelecidas para solução de questões de saúde urgentes serão permitidos pelas Comissões de Ética desde que os requisitos éticos na condução dos ensaios clínicos não sejam diminuídos, sendo considerada urgente a disponibilidade de produtos eficazes que já existam a todos que deles necessitem.

Considerando as diretrizes éticas avaliadas, 88,5\% continham a justificativa do uso do placebo e os demais artigos (11,5\%) não justificavam, pois, tanto a metodologia utilizada, como os resultados, não foram subsídios suficientes para tal ${ }^{(40)}$.

(38) LEWIS, J. et al., op. cit., p.1337-1340.

(39) LIMA, C. Ensaios clínicos: vulnerabilidade e relativismo ético. Acta Medica Portuguesa, Lisboa, v. 18, p. 221-226, 2005. Disponível em: <http://www.actamedicaportuguesa.com/pdf/2005-18/3/ 221-226.pdf>. Acesso em: 20 mar. 2007.

(40) EMANUEL, E. J.; MILLER, F. G. The ethics of placebo-controlled trials-a middle ground. The New England Journal of Medicine, Boston, v. 345, n. 12, p. 915-919, sept. 2001. 
No período de 2002 a 2004, foram analisados 636 projetos, pelo CONEP, sendo 42 reprovados. Entre esses, 7 (16,6\%) não continham razão ética para o uso de placebo, ou washout (justificativa inaceitável)(40). Sugere-se que, pelo mesmo motivo de reprovação aplicado pelo Conep, 25 artigos $(11,5 \%)$ não deveriam ter sido aprovados por Comitê de Ética, em primeira análise, pois implica na não observância da legislação e na não proteção do sujeito de pesquisa. No entanto, há de se considerar que nenhuma norma ou lei vai impedir eventuais abusos em pesquisa e problemas de natureza ética são os principais motivos de reprovação de projetos, incluindo o uso do placebo $^{(42)}$.

\section{CONSIDERAÇÕES FINAIS}

$\mathrm{Na}$ análise dos artigos científicos, observaram-se informações limitadas sobre as pesquisas, dificultando assim a avaliação ética do experimento. A forma de edição dos artigos científicos também interferiu no trabalho, não contendo a justificativa para o uso do placebo.

A conduta ética de todas as pesquisas realizadas deve estar presente não somente entre os responsáveis pelo estudo, mas, também entre as agências reguladoras, patrocinadores de pesquisa, instituições de revisão e editores de revistas, que devem sempre contestar o uso do placebo e exigir explicação para seu uso em cada protocolo e edição da pesquisa, evitando que estudos não-éticos sejam divulgados ${ }^{(43)}$.

A repercussão da Resolução CNS n. 196/96 foi verificada segundo suas diretrizes e conclui-se o cumprimento em vários aspectos, mas, não no total das edições, indicando o não cumprimento da norma em pelo menos $11,5 \%$ dos casos.

Diante das disposições e resultados apresentados, conclui-se que a maioria dos ensaios clínicos realizados no Brasil respeita a Resolução CNS n. 196/96 ${ }^{(44)}$. Porém, ainda há a necessidade de maior rigor no cumprimento dos preceitos éticos quanto ao uso de grupo controle-placebo que promoverão a proteção devida aos sujeitos da pesquisa. Além disso, cuidados por parte dos pesquisadores e do Comitê de Ética devem ser intensificados, pois, existem estudos que não justificam o uso do placebo.

(41) FREITAS, C. B. D.; LOBO, M.; HOSSNE, W. S., op. cit.

(42) SILVA, J. C. Para Conep, só norma não impede abuso de pesquisas com humanos. Folha de $S$. Paulo, São Paulo, 29 jun. 2001.

(43) ROTHMAN, K. J.; MICHELS, K. B. The continued unethical use of placebo controls. The New England Journal of Medicine, Boston, v. 331, n. 6, p. 394-398, 1994.

(44) BRASIL. Ministério da Saúde. Conselho Nacional de Saúde. Resolução n. 196/1996, de 10 de outubro de 1996. Diretrizes e normas regulamentadoras sobre pesquisa envolvendo seres humanos, cit. 


\section{REFERÊNCIAS BIBLIOGRÁFICAS}

ACADEMIA BRASILEIRA DE REUMATOLOGIA. A Produção Científica Brasileira. Boletim Acadêmico - ABR, Recife, v. 6, n. 1, p. 4-5, jan./mar. 2006. Disponível em: <http://www.academiareumatol.com.br/boletim/boletim11.pdf>. Acesso em: 20 nov. 2007.

AMDUR, R. J.; BIDDLE, C. Institutional review board approval and publication of human research results. JAMA, Chicago, v. 277, n. 11, p. 909-914, 1997.

BIREME/OPAS/OMS. Biblioteca Virtual em Saúde e o controle bibliográfico da produção científica da América Latina e Caribe: o sistema e bases de dados LILACS. São Paulo: Bireme, 2006. Disponível em: <http:// bvsmodelo.bvsalud.org/site/Lilacs>. Acesso em: 30 maio 2006.

BRASIL. Ministério da Saúde. Conselho Nacional de Saúde. Resolução $n$. 196/1996, de 10 de outubro de 1996. Diretrizes e normas regulamentadoras sobre pesquisa envolvendo seres humanos. Brasília: Conselho Nacional de Saúde, 1996. Disponível em: <http://conselho.saude.gov.br/comissao/conep/ resolucao.html>. Acesso em: 20 nov. 2007.

Ministério da Saúde. Agência Nacional de Vigilância Sanitária. Considerações e definições para Pesquisa Clínica. [s. d.]. Disponível em: <http:/ /www.anvisa.gov.br/medicamentos/pesquisa/def.htm>. Acesso em: 20 mar. 2006.

COMISSÃO NACIONAL DE ÉTICA EM PESQUISA. A CONEP responde: Três questões básicas. Cadernos de Ética em Pesquisa, Brasília, v. 1, n. 2, p. 4-6, 1998.

CONFERÊNCIA INTERNACIONAL DE HARMONIZAÇÃO. Manual para boas práticas clínicas: versão Harmonizada Tripartite (USA, Europa e Japão). Tradução de Marion Roussel, 1996. Disponível em: <http://www.ufrgs.br/bioetica/gcpport.htm>. Acesso em: 30 maio 2006.

EMANUEL, E. J.; MILLER, F. G. The ethics of placebo-controlled trials-a middle ground. The New England Journal of Medicine, Boston, v. 345, n. 12, p. 915-919, sept. 2001.

FORTES, P. A. C. As pesquisas em seres humanos. Cadernos de Ética em Pesquisa, Brasília, v. 1, n. 2, p. 22, 1998.

FREITAS, C. B. D.; LOBO, M.; HOSSNE, W. S. Oito anos de evolução: um balanço do Sistema CEP-CONEP. Cadernos de Ética em Pesquisa, Brasília, v. 6, n. 16, p. 20-30, 2005. Disponível em: <http://conselho.saude.gov.br/comissao/conep/publicacoes_cep.html>. Acesso em: 20 nov. 2007.

GUIMARÃES, J. A. A pesquisa médica e biomédica no Brasil: comparações com o desempenho científico brasileiro e mundial. Ciência \& Saúde Coletiva, Rio de Janeiro, v. 9, n. 2, p. 303-327, 2004. 
HOSSNE, W. S.; VIEIRA, S. Pesquisas com cooperação estrangeira: qual cooperação? Cadernos de Ética em Pesquisa, Brasília, v. 6, n. 14, p. 3-5, 2004. [editorial]

LEITE, F.; SILVA, J. C. Em nome da ciência: Brasil já tem 500 mil "cobaias" humanas. Folha de S. Paulo, São Paulo, 29 jun. 2001.

Para Conep, só norma não impede abuso de pesquisas com humanos. Folha de S. Paulo, São Paulo, 29 jun. 2001.

LEWIS, J. et al. Placebo-controlled trials and the Declaration of Helsinki. Lancet, London, v. 359, n. 9314, p. 1337-1340, 2002.

LIMA, C. Ensaios clínicos: vulnerabilidade e relativismo ético. Acta Medica Portuguesa, Lisboa, v.18, p.221-226, 2005. Disponível em: <http:// www.actamedicaportuguesa.com/pdf/2005-18/3/221-226.pdf>. Acesso em: 20 mar. 2007.

OLIVEIRA, G. G. Ensaios clínicos: princípios e práticas. Brasília: Ministério da Saúde, 2006. p. 255-256.

PELLIZZON, R. F.; POBLACIÓN, D.A.; GOLDENBERG, S. Pesquisa na área da saúde: seleção das principais fontes para acesso à literatura científica. Acta Cirurgica Brasileira, v. 18, n. 6, 2003.

PINTO, M. Relatório setorial final: setor farmacêutico - Lab. Nacionais. Rede DPP FINEP, 2004. Disponível em: <http://www.finep.gov.br/PortalDPP>. Acesso em: 20 abr. 2007.

ROTHMAN, K. J.; MICHELS, K. B. The continued unethical use of placebo controls. The New England Journal of Medicine, Boston, v. 331, n. 6, p. 394-398, 1994.

SARDENBERG, T. et al. Análise dos aspectos éticos da pesquisa em seres humanos contidos nas Instruções aos autores de 139 revistas científicas brasileiras. Revista da Associação Médica Brasileira, São Paulo, v.45, n.4, p.295-302, 1999.

Análise dos aspectos éticos da pesquisa em seres humanos contidos nas instruções aos autores de 38 revistas de ortopedia e traumatologia. Acta Ortopedica Brasileira, São Paulo, v. 10, n. 2, p. 15-18, 2002.

TRIALS of war criminals before the Nuremberg military tribunals under Control Council Law n. 10. Washington: Government Printing Office, v. 2, p. 181-182, 1949.

VERSIANI, M. A necessidade do Grupo-Controle com placebo em pesquisas sobre a eficácia de tratamentos psiquiátricos. Revista Bioética, Brasília, v. 8 , n. 1, p. 29-42, 2000. 
VILARDAGA, V. Autorização para testes clínicos com brasileiros cresce 10\% ao ano. Revista Pesquisa FAPESP, São Paulo, n. 83, jan. 2003. Disponível em: <http://www.revistapesquisa.fapesp.br>. Acesso em: 20 abr. 2007.

ZORZETTO, R. et al. The scientific production in health and biological sciences of the top 20 Brasilian Universities. Brazilian Journal of Medical and Biological Research, São Paulo, v. 39, n. 12, p. 1513-1520, 2006.

WORLD MEDICAL ASSOCIATION. Declaration of Helsinki. Recommendation guiding physicians in biomedical research involving humans subjects. JAMA, Chicago, v. 277, n. 11, p. 925-926, Mar. 1997. 